\title{
Tiempo de desarrollo y supervivencia de Arhopalus syriacus (Reitter) (Coleoptera: Cerambycidae), una plaga potencialmente perjudicial de Pinus sp. en Argentina
}

\author{
Fachinetti, R.; M.L. Pedemonte y M.P. Grilli
}

\begin{abstract}
RESUMEN
Arhopalus syriacus es una especie exótica presente en los pinares de Argentina que ataca especies de Pinus. El objetivo de este trabajo fue determinar el tiempo de desarrollo y la supervivencia de $A$. syriacus a $25^{\circ} \mathrm{C}$ usando dieta artificial. Tiempos, tasas de desarrollo, pesos, medidas de cápsulas cefálicas y largo de las larvas fueron observados en condiciones de laboratorio; mientras que la proporción de sexos, emergencia y reproducción de los adultos se registraron utilizando trozas de pinos. Las larvas puparon después de 6, 7, 8 y 9 estadios. El ancho de las cápsulas cefálicas y el largo de las larvas disminuyeron a partir del cuarto y sexto estadio, respectivamente. Las pupas hembra fueron más grandes y pesadas. La supervivencia fue menor en los primeros dos estadios y llegó al 100\% en larvas maduras y pupas. Las máximas abundancias de adultos que emergieron de las trozas de pino ocurrieron en los meses de noviembre y diciembre. Los machos adultos vivieron 9,27 $\pm 0,84$ días y fueron más livianos que las hembras, que vivieron $9,12 \pm 1,61$ días. El número de larvas por hembra fue $80,65 \pm 10,62$. El ciclo de vida total de A. syriacus duró 298,86 \pm 13,66 días.
\end{abstract}

Palabras clave: Arhopalus syriacus, Pinus sp., desarrollo, supervivencia, estadios larvales

Fachinetti, R.; M.L. Pedemonte and M.P. Grilli, 2015. Development time and survival of Arhopalus syriacus (Reitter) (Coleoptera: Cerambycidae), a potential pest of Pinus sp. in Argentina. Agriscientia 32 (2): 95-105

\section{SUMMARY}

Arhopalus syriacus is an exotic longhorn beetle that attacks mainly Pinus and is present in pine forests of Argentina. The objective of this study was to estimate development and survival of $A$. syriacus at $25{ }^{\circ} \mathrm{C}$ on an artificial diet. Developmental time, developmental rates, pupal weights, larval length, and head capsule widths were recorded in laboratory conditions whereas emergency time of adults, sex ratio and female reproduction were studied using pine logs. Larvae pupated after 6, 7, 8 and 9 stadia. Head-capsule width and larval length decreased from the fourth and sixth instar respectively. Female pupae were heavier than male ones and this situation was the same for pupal 
length. Survival was lower in the first two instars but increased to $100 \%$ in all mature larvae and pupae. The maximum abundances of adults from pine logs occurred in November and December. Male adults lived $9.27 \pm 0.84$ days and female $9.12 \pm 1.61$ days. Female adults were heavier than males and the number of new larvae per female was $80.65 \pm 10.62$. The life cycle duration of A. syriacus was $298.86 \pm 13.66$ days.

Key words: Arhopalus syriacus, Pinus sp., development, survival, larval instar

R. Fachinetti, M.L. Pedemonte y M.P. Grilli: Centro de Relevamiento y Evaluación de Recursos Agrícolas y Naturales (CREAN-IMBIV); Av. Valparaíso S/N, Facultad de Cs. Agropecuarias, Universidad Nacional de Córdoba, CC 509-5000, Córdoba, Argentina. Correspondencia a: rominafachinetti@gmail.com

\section{INTRODUCCIÓN}

Los escarabajos de la madera de la familia Cerambycidae (Polyphaga: Chrysomeloidea) constituyen un grupo relevante del orden Coleoptera, representado por alrededor de 35.000 especies en el mundo (Minelli, 1993).

En términos ecológicos, estos insectos propician las condiciones para la invasión de hongos descomponedores de la madera, y su actividad resulta fundamental en el proceso de la circulación de minerales hacia el suelo. Sin embargo, también son considerados plaga, ya que pueden dañar la integridad de árboles vivos llegando a provocar su muerte, reducir la calidad de vigas o maderas estructurales y funcionar como vectores de enfermedades (Ciesla, 2011; Wang et al., 2014).

El género Arhopalus (Cerambycidae: Aseminae), oriundo del Hemisferio Norte, se encuentra presente en las principales regiones biogeográficas del mundo como consecuencia del comercio de madera (Bense, 1995) y la falta de control de los países importadores. Como resultado de esto, se ha establecido en nuevas localidades donde la ausencia de enemigos naturales, entre otros factores, ha favorecido el aumento de sus poblaciones alcanzando el status de plaga (Ciesla, 2011).

Las especies de Arhopalus han sido registradas atacando principalmente representantes de Pinus y Picea (Bense, 1995) y también se encuentran asociadas a otras coníferas, como Abies, Cedrus, Cryptomeria, Cupressus, Juniperus y Pseudotsuga (Brown, 1968; Duffy, 1968; Hosking \& Bain, 1977). Actualmente, este género se presenta como vector del nemátodo Bursaphelenchus xylophilus (Robertson et al., 2008; Wang et al., 2014).

En la Argentina, Di lorio (2004), registró por primera vez al género Arhopalus en la ciudad de Buenos Aires.

Arhopalus syriacus (Reitter, 1895) es nativo de la región oriental del Mediterráneo (Brown, 1968) y está presente en Italia, Chipre y Australia Occidental (Martelli, 1952; Duffy, 1963; Brown, 1968). En Córdoba, A. syriacus fue detectada en 2007 en la localidad de Alpa Corral, emergiendo de Pinus elliottii, previamente infestadas por Sirex noctilio (Hymenoptera: Siricidae) (López et al., 2008). La asidua presencia de este cerambícido en pinos con síntomas de grave deterioro, suscitaron la duda sobre su papel real en la dinámica del bosque como descomponedor en tocones y árboles muertos, para ser considerado una potencial plaga forestal (Iglesias, 2009).

En los bosques de coníferas, el daño principal es causado por las larvas de $A$. syriacus, que barrenan el floema y la superficie adyacente a la madera, formando profundas e intricadas galerías (Brown, 1968). Como otros cerambícidos, se alimentan del cambium (Coulson \& Witter, 1990) originando grandes orificios y túneles en la madera que producen niveles significativos de degradación de los productos forestales (Safranyik \& Raske, 1970; Cerezke, 1977). Al permanecer extensos periodos en el interior de la madera, las larvas pueden ser transportadas largas distancias dentro de troncos, embalajes, pallets o leña.

Cuando los adultos emergen de los troncos, dejan orificios de salida de forma ovalada, lo que facilita la identificación de los daños causados (Wallace, 1954; Hosking \& Bain, 1977; Brockerhoff \& Hosking, 2001).

Los adultos de $A$. syriacus miden entre 11,4 y 22,1 mm de largo, presentan tegumento marrón amarillento a marrón oscuro, el segmento terminal 
del palpo maxilar expandido y triangular (con un largo igual o mayor que su ancho apical) y el III tarsito inciso casi hasta la base. Los élitros poseen ángulos suturales redondeados y en los machos el VIII tergito es levemente emarginado en el ápice (Wang \& Leschen, 2003). A diferencia de otras especies del género, A. syriacus posee un rango de distribución estrecho y se halla especializado en el género Pinus sp. (Bense, 1995; Webb \& Eldridge, 1997). Ataca pinos debilitados o muertos por el fuego $u$ otras causas y no se han encontrado evidencias de que afecten árboles sanos (Webb \& Eldridge, 1997). En la provincia de Córdoba, Pedemonte (2014) registró a A. syriacus atacando ejemplares de $P$. taeda.

Cada hembra adulta de Arhopalus puede llegar a colocar más de 1.000 huevos y el periodo de oviposición se extiende a lo largo de su vida, desde pocos días a casi tres semanas. Los huevos miden entre 0,5 y $1,8 \mathrm{~mm}$, son opacos, blancos, con forma de cigarro y colocados en grupos de 1 a 50 (Hosking \& Bain, 1977).

A pesar de su importancia como plagas forestales, poco se conoce acerca de la dinámica poblacional y ecología de las especies de Arhopalus presentes en la Argentina, posiblemente debido a que no han sido consideradas de importancia en las dinámicas de los bosques ni tampoco como potenciales plagas alrededor del mundo. Información sobre la biología de $A$. syriacus ha sido provista por Martelli (1952), Moore (1962, 1963), Duffy (1963) y Brown (1968); sin embargo, no se conocen tiempos de desarrollo ni supervivencia de la especie. Por ello, el objetivo de este trabajo se centró en determinar los tiempos de desarrollo de los estados y estadios larvales y la supervivencia de A. syriacus a $25^{\circ} \mathrm{C}$ usando dieta artificial.

\section{MATERIALES Y MÉTODOS}

\section{Área de estudio}

El estudio se desarrolló en el suroeste de la provincia de Córdoba, Argentina, en el área comprendida entre los $32^{\circ} 33^{\prime}$ y $32^{\circ} 35^{\prime} \mathrm{S}$ y $64^{\circ} 43^{\prime}$ y $64^{\circ} 37^{\prime}$ $\mathrm{O}$. En esta región se encuentra una importante área forestada con pinos, donde las especies forestales más abundantes son Pinus elliottii y P. taeda.

\section{Árboles trampa}

Para determinar los periodos de emergencia y las proporciones de sexos de A. syriacus, se utilizaron trozas de pinos de árboles trampa: pinos previamente debilitados mediante la aplicación del herbicida Banvel ${ }^{\circledR}$ (Dicamba $48 \%$. Dosis $1 \mathrm{ml} / 10 \mathrm{~cm}$ de perímetro basal), provenientes de ocho parcelas ubicadas en la zona de estudio.

Cada parcela constó de cinco árboles trampa en septiembre/octubre del 2012. En junio de 2013 se seleccionaron y apearon dos de estos árboles por parcela, que fueron dejados en el lugar hasta octubre, donde se procedió al corte de las trozas, de un metro de largo. Estas fueron llevadas al laboratorio y colocadas en jaulas entomológicas, las que fueron revisadas cada 48-72 hs (Pedemonte, 2014). Los adultos que emergieron de ellas fueron clasificados por fecha de emergencia, sexo y especie.

Para conocer la proporción de sexos de los adultos emergidos de las trozas de árboles trampa, se compararon las abundancias medias mensuales de cada sexo para todas las parcelas del área de estudio. También, se registraron los días de vida y el peso al morir (mg) de estos adultos. Además, se determinó el periodo de preoviposición de las hembras (desde el día de emergencia del adulto hasta la primera ovipostura) y el número de larvas emergidas provenientes de hembras de diferente condición (hembras en pareja y hembras solas) (Fachinetti, 2012).

Los datos resultantes de los árboles trampa fueron analizados mediante la prueba de Kruskal Wallis, debido a que no cumplieron con el supuesto de normalidad (estadístico de Shapiro-Wilks, modificado por Mahibbur \& Govindarajulu, 1997).

\section{Cría y mantenimiento de Arhopalus syriacus en condiciones de laboratorio}

Para iniciar la cría de A. syriacus en laboratorio, en noviembre y diciembre del 2013 , se seleccionaron 42 hembras adultas y 22 machos procedentes de las jaulas entomológicas. Se formaron 22 parejas, mientras que las 20 hembras restantes se colocaron solas (sin el macho adulto). Los adultos permanecieron en un recipiente plástico $(6 \mathrm{~cm} \times 4 \mathrm{~cm}$ de diámetro), con un trozo de corteza de pino que sirvió como sostén a las oviposturas. Los trozos de corteza fueron observados diariamente para percibir la presencia de huevos. Todos los huevos permanecieron en el mismo recipiente plástico que los adultos hasta que se observaron larvas. Las larvas eclosionadas, ( $\leq 12$ hs de vida), se colocaron individualmente en cápsulas de Petri $(6 \mathrm{~cm}$ de diámetro) con dieta artificial semi-sintética (Iglesias et al., 1989). Las cápsulas de Petri fueron ubicadas en bandejas plásticas $(28 \mathrm{~cm} \times 38 \mathrm{~cm} \times 9 \mathrm{~cm}$ de alto, aproximadamente) y llevadas a una incubadora programada a $25^{\circ} \mathrm{C} \pm 1{ }^{\circ} \mathrm{C}$ (LIADE Bambi 
Modelo CTL-V3.11). Se dispuso de 100 larvas para compensar la eventual mortalidad y lograr un mínimo de al menos 20 individuos finales. El cambio de dieta artificial, higiene de las cápsulas de Petri y las mediciones de los individuos se realizaron cada 15 días.

Las larvas de $A$. syriacus permanecieron en las cápsulas de Petri hasta llegar al estado de pupa. Las pupas fueron pesadas (mg) y medidas (largo en $\mathrm{cm}$ ) y se colocaron en tacos perforados de madera de pino $(5 \mathrm{~cm} \times 5 \mathrm{~cm} \times 5 \mathrm{~cm})$ hasta la emergencia del individuo adulto, a los que se les determinó el sexo.

\section{Desarrollo y supervivencia}

Los tiempos de desarrollo fueron establecidos mediante el registro de la duración, en días: desde la oviposición hasta la emergencia de la larva I (estado de huevo), desde la emergencia de la larva hasta la formación de la pupa (estado de larva) y desde el primer día como pupa hasta la emergencia del adulto (estado de pupa). Se registró, además, el tiempo de vida de los adultos. A partir de los datos de tiempo de desarrollo (en días), se obtuvieron las tasas de desarrollo (1/días) y su error asociado (error estándar) para cada estado y estadios larvales.

El número de estadios larvales y su tiempo de desarrollo fueron registrados. Para ello se observaron minuciosamente las cápsulas de Petri donde se criaron los individuos, para detectar la presencia de exuvias y restos de cápsulas cefálicas, delimitando así el cambio de un estadio a otro. Además, se registraron el ancho de las cápsulas cefálicas $(\mathrm{mm})$ y el largo del cuerpo de las larvas (cm).

La supervivencia de $A$. syriacus se registró mediante el conteo de individuos presentes en cada estado y estadio de desarrollo. La proporción de sobrevida del estado de huevo no se registró debido a que la cría de $A$. syriacus comenzó desde que se percibieron larvas en los recipientes que contenían a la pareja de adultos. Los datos de supervivencia fueron tabulados y analizados por medio del test de Kaplan-Meier (Altman, 1991). Se obtuvieron así las curvas de supervivencia para cada estado y estadio de desarrollo.

\section{RESULTADOS Y DISCUSIÓN}

\section{Árboles trampa}

En total se registró la emergencia de 510 individuos adultos de $A$. syriacus provenientes de trozas de pinos de las parcelas de árboles trampa, desde el 28/10/13 hasta el 26/11/2014 (Figura 1). Se observó que los orificios de salida de los adultos fueron ovalados, de 6 a $7 \mathrm{~mm}$ de alto y con orientación paralela al eje longitudinal del tronco, datos que concuerdan con los resultados de Ciesla (2011) para cerambícidos que atacan madera y con los obtenidos para A. ferus por Hosking y Bain (1977).

El periodo de máxima actividad de los adultos de A. syriacus abarcó desde la primavera (104 hembras y 105 machos) hasta fines del verano (55 hembras y 67 machos), tal como ha sido registrado para otras especies de Arhopalus (Wallace, 1954; Duffy, 1968; Bense, 1995; Webb \& Eldridge, 1997; Brockerhoff \& Hosking, 2001; Pedemonte, 2014). Las emergencias registradas en octubre y noviembre de 2014, pudieron deberse a la re-infección de las trozas por parte de las hembras emergidas al principio de la campaña.

La abundancia media mensual de machos fue ligeramente mayor que la de hembras, aunque esta diferencia no fue significativa ( $p>0,05)$ (Fig.1). En el pico del periodo de actividad de los adultos, el número de machos superó al de hembras. Esto se debe a que los machos emergen unos días antes para fecundar a las hembras recién emergidas, observación que coincide con lo registrado para A. ferus y A. rusticus (Hosking \& Bain, 1977; Hutcheson, 1980; Pedemonte, 2014). Esto explica también que las hembras sin pareja dejaron descendencia porque fueron fecundadas dentro de las jaulas entomológicas antes de ser llevadas al laboratorio.

Las hembras adultas emergidas de los árboles trampa vivieron 9,12 \pm 0,61 días, en concordancia con lo reportado por Hosking y Bain (1977), quienes registraron para hembras de $A$. ferus un periodo comprendido entre unos pocos días a 3 semanas, a $4{ }^{\circ} \mathrm{C}$. Los machos vivieron 9,27 $\pm 0,84$ días. Al comparar los días de vida de los adultos de $A$. syriacus, no presentaron diferencias significativas entre sexos ( $N=64 ; H=2,0 E-04 ; p=0,9886)$. El peso de las hembras adultas $(67,63 \pm 3,13 \mathrm{mg})$ fue significativamente mayor ( $N=64 ; \mathrm{H}=9,67 ; \mathrm{p}=0,0019)$ que el de los machos $(48,25 \pm 4,32 \mathrm{mg})$.

Las variables días de vida, periodo de preoviposición (días) y peso al morir (mg), entre hembras que se encontraban solas con respecto a las que fueron colocadas en pareja, no mostraron diferencias significativas $(p>0,05)$ (Tabla 1). Sin embargo, la abundancia de larvas eclosionadas fue mayor en las hembras que se encontraban solas con respecto a las que tenían pareja (Tabla 1). Este comportamiento se atribuye al posible estrés ocasionado por la presencia del macho en el mismo recipiente (Larsson, 2010). 


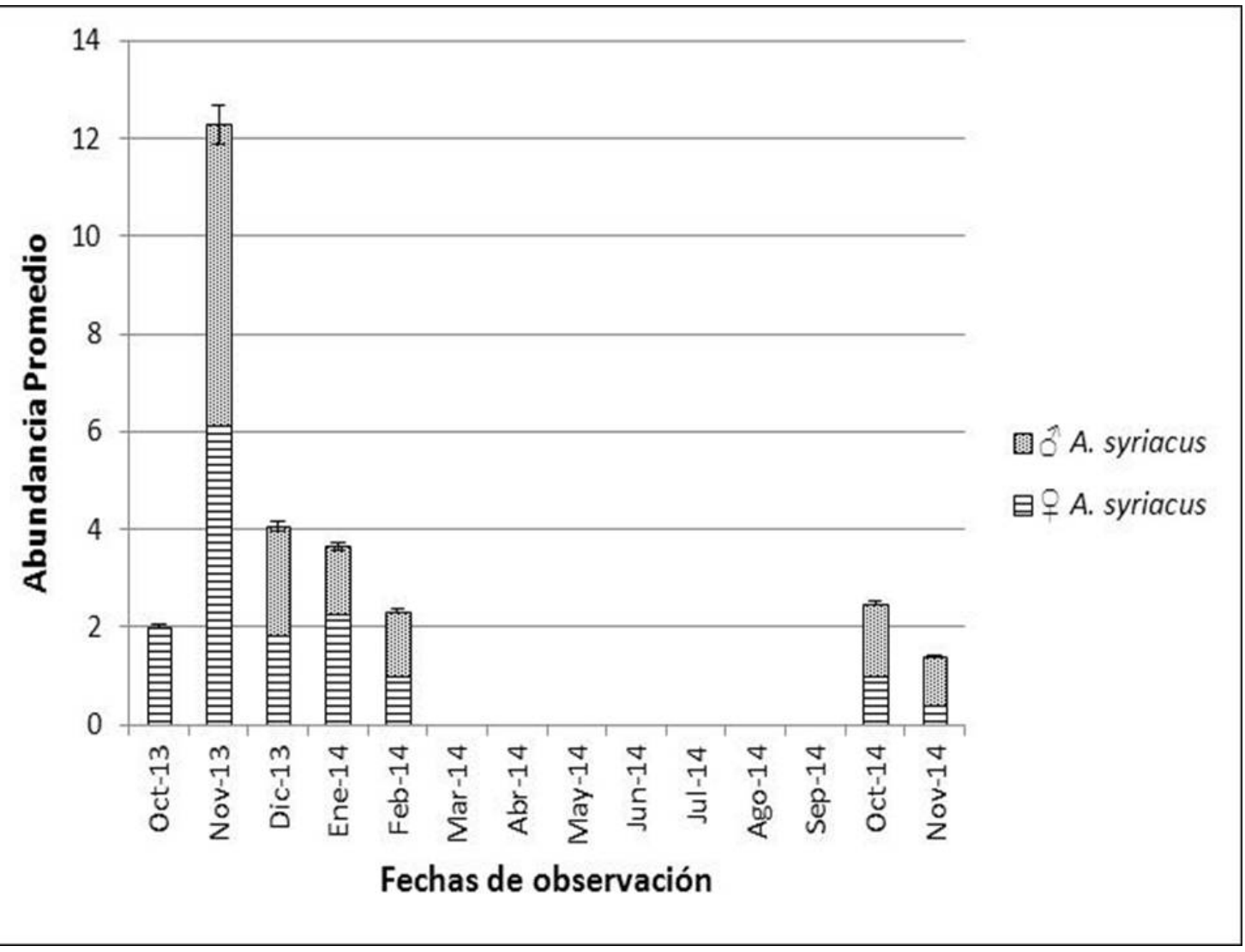

Figura 1. Distribución temporal de la abundancia promedio de adultos de A. syriacus emergidos de "árboles trampa".

Tabla 1. Días de vida, preoviposición (días), peso al morir (mg) y número de larvas eclosionadas de adultos hembra de Arhopalus syriacus bajo diferente condición (solas o con pareja) (Promedio \pm E.E.).

\begin{tabular}{ccccc}
\hline Condición & Días de vida & Preoviposición & Peso al morir & Número de larvas \\
\hline O Solas (20) & $9,00 \pm 0,83 \mathrm{a}^{2}$ & $4,45 \pm 0,82 \mathrm{a}$ & $70,86 \pm 4,75 \mathrm{a}$ & $80,65 \pm 10,62 \mathrm{a}$ \\
9 con Pareja (22) & $9,23 \pm 0,79 \mathrm{a}$ & $5,41 \pm 0,79 \mathrm{a}$ & $64,70 \pm 4,53 \mathrm{a}$ & $37,77 \pm 10,12 \mathrm{~b}$ \\
& $\mathrm{H}^{3}=0,08$ & $\mathrm{H}=0,71$ & $\mathrm{H}=0,75$ & $\mathrm{H}=7,96$ \\
& $\mathrm{p}=0,7691$ & $\mathrm{p}=0,3874$ & $\mathrm{p}=0,3849$ & $\mathrm{P}=0,0046$ \\
\hline
\end{tabular}

1. Los números entre paréntesis representan la cantidad de individuos.

2. Medias con una letra minúscula común no son significativamente diferentes entre hembras de distinta condición $(p>0,05)$.

3. Los valores de $\mathrm{H}$ y $\mathrm{p}$ representan el análisis de Kruskal-Wallis.

\section{Desarrollo y supervivencia}

De la cría de A. syriacus en laboratorio mediante dieta artificial, 21 individuos completaron su ciclo llegando al estado adulto: 11 hembras y 10 machos. El tiempo de desarrollo promedio de los estados de huevo, larva, pupa y adulto fue de 12,10 $\pm 0,40 ; 252,29 \pm 13,72 ; 16,38 \pm 0,53$ y $18,10 \pm 0,69$ días, respectivamente. La duración total del ciclo fue de 298,87 \pm 13,66 días, resultado menor que el obtenido por Hosking y Bain (1977), en donde
A. ferus completó su desarrollo en 1 a 2 años para un rango térmico de 4 a $29,4^{\circ} \mathrm{C}$. En el presente estudio, la finalización del período de desarrollo total bajo condiciones controladas de temperatura, coincidió con el inicio del período de emergencia observado a campo (primavera).

Los machos adultos vivieron $18,90 \pm 1,00$ días promedio, mientras que las hembras 17,36 \pm 0,95 días; no se encontraron diferencias estadísticamente significativas $(N=21 ; H=1,52 ; p=0,2121)$ en- 
tre sexos. Los valores de las tasas de desarrollo promedio para cada estado (huevo, larva, pupa y adulto) y estadio larval (larva I, larva II, larva III, larva IV, larva V, larva VI, larva VII, larva VIII y larva IX) se representan en la Figura 2. Las tasas de desarrollo de los estados de huevo y pupa, son similares con los reportados por Pershing y Linit (1986) para Monochamus carolinensis (Coleoptera: Cerambycidae) a $38^{\circ} \mathrm{C}$. Sin embargo, Keena y Moore (2010) obtuvieron valores mayores en las tasas de desarrollo de los estadios larvales de Anoplophora glabripennis (Coleoptera: Cerambycidae) criada con dieta artificial para un rango térmico de $15 \mathrm{a}$ $30^{\circ} \mathrm{C}$.

En lo que respecta al desarrollo de los estados inmaduros, la larva de A. syriacus eclosionó luego de 12 días, coincidente con lo reportado por Hosking y Bain (1977) para individuos de A. ferus criados dentro de troncos, en un rango térmico de 4 a $29,9^{\circ} \mathrm{C}$.

Las larvas de A. syriacus puparon luego de 6, 7, 8 o 9 estadios: 5 individuos culminaron su desarro- llo larval luego de 6 estadios, 10 después de 7, 4 luego de 8 y 2 después de 9 estadios. Al comparar el tiempo de desarrollo del estado de larva entre los individuos con diferente número de estadios larvales, no se registraron diferencias significativas $(H=5,40 ; N=21 ; p=0,1444)$ (Figura 3). El tiempo de desarrollo del estado de larva para los individuos de 6, 7, 8 y 9 estadios fue de $297 \pm 25,88 ; 273,8$ $\pm 18,3 ; 324,28 \pm 28,93 ; 378 \pm 40,92$ días, respectivamente. La diferencia en el tiempo de desarrollo del estado de larva para los individuos de 6, 7, 8 y 9 estadios fue de un mes aproximadamente, que coincide con los cuatro periodos de emergencia de los adultos procedentes de los árboles trampa.

El tiempo de desarrollo de larva III fue significativamente diferente entre las larvas que tuvieron 6 y 9 estadios ( $\mathrm{N}=21 ; \mathrm{H}=8,72 ; \mathrm{p}=0,0330$ ) (Fig. 3). No se observaron diferencias significativas al comparar el último tiempo de desarrollo entre larvas de 6 , 7 y 8 estadios ( $N=19 ; \mathrm{H}=14,92 ; \mathrm{p}>0,0001$ ) (Fig. 3).

El número de estadios larvales (larva I, larva II, larva III, larva IV, larva V, larva VI, larva VII, larva VIII

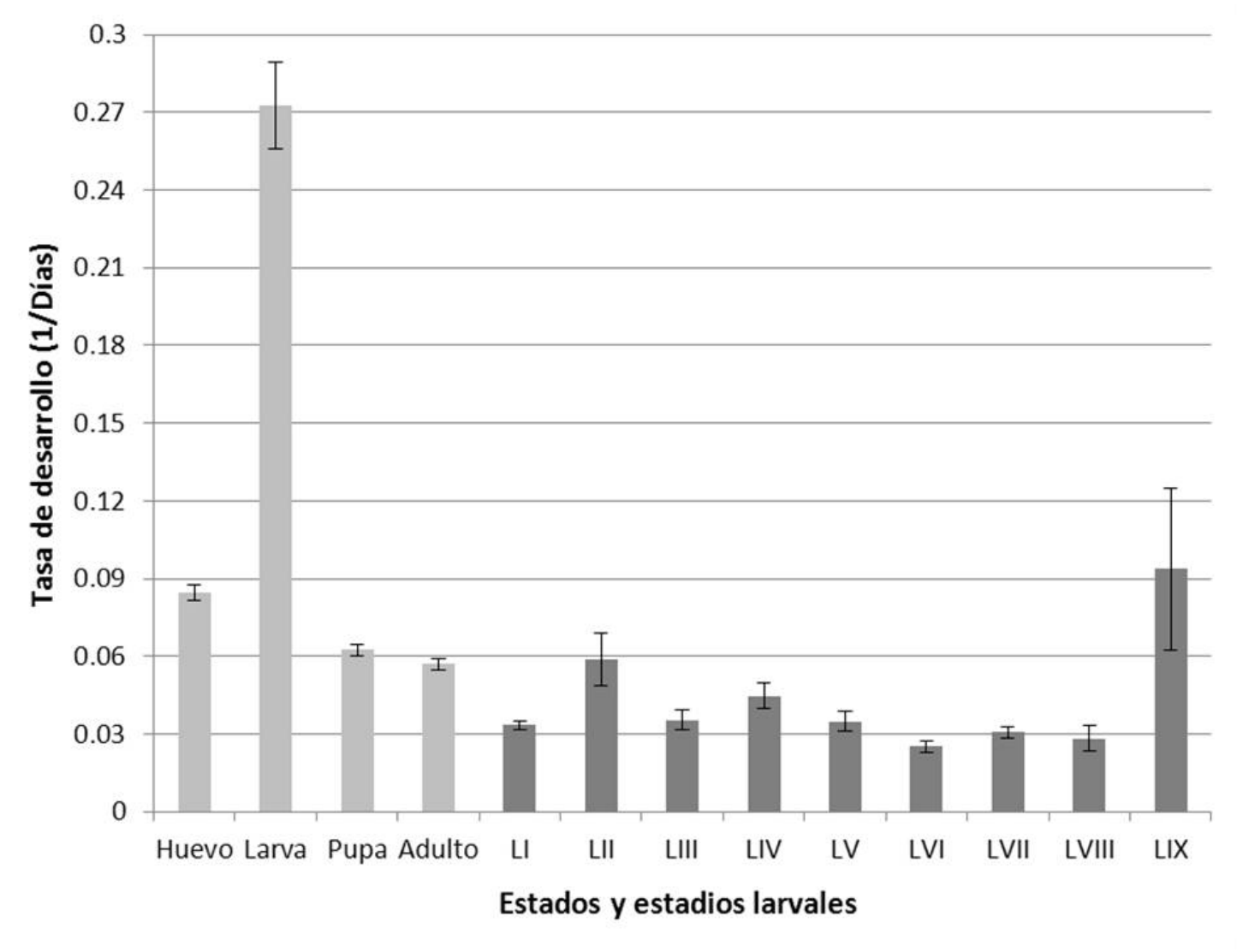

Figura 2. Tasas de desarrollo promedio (1/días de desarrollo) y error asociado (E.E.) de los estados y estadios larvales de A. syriacus. 


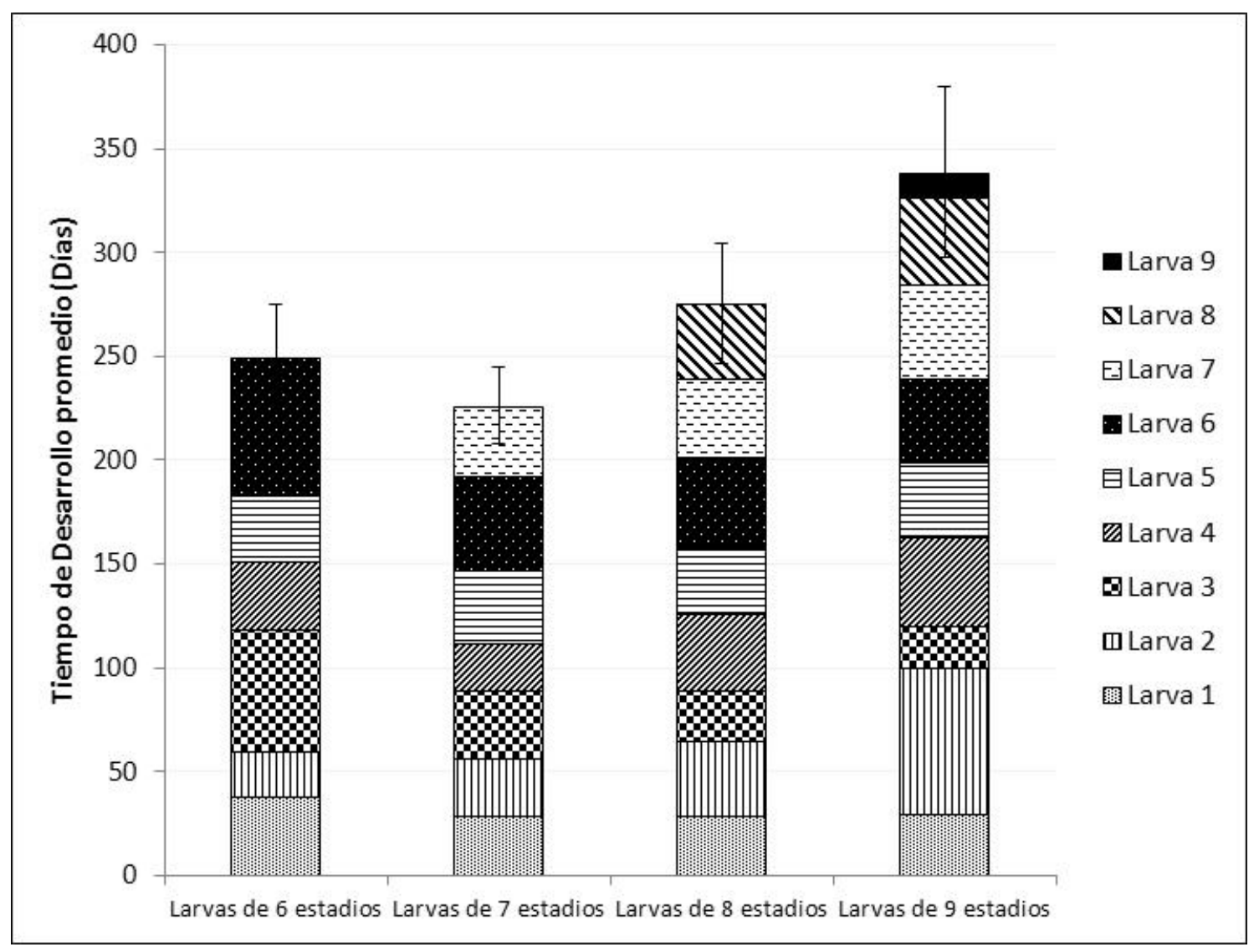

Figura 3. Tiempo de desarrollo promedio del estado de larva de A. syriacus para individuos que terminaron su desarrollo larval en diferentes estadios.

y larva IX) de A. syriacus obtenido en este trabajo, concuerda con lo registrado por Kenna y Moore (2010) para Anoplophora glabripennis. Sin embargo, el número de estadios larvales registrados fue mucho menor que los reportados por Rose (1957) para el cerambícido Monochamus scutellatus y por Naves et al. (2008) para M. galloprovincialis criados a temperatura ambiente utilizando troncos de pino.

En lo que respecta a $A$. syriacus, no se encontraron antecedentes de estudios en laboratorio donde se presentara esta variabilidad intraespecífica en el números de estadios larvales. En este sentido, los datos aquí presentados aportan los primeros resultados y señalan que a condiciones óptimas de temperatura (Esperk et al., 2007), el desarrollo del estado larva de $A$. syriacus puede completarse después de 6 estadios. Al madurar antes, $A$. syriacus podría desarrollar una generación adicional (Gomi, 1996; Fischer \& Fiedler, 2001; Gomi et al. 2003). El valor adaptativo de la variabilidad en el número de estadios larvales está en alcanzar o mantener la etapa de desarrollo frente a diferentes condiciones ambientales, en la forma de omitir o insertar estadios (Esperk, 2006).

Se estimaron promedios y límites de clase para cada estadio larval (Tabla 2). Se observaron diferencias estadísticamente significativas entre las variables ancho de cápsulas cefálicas y largo de las larvas comparando los 9 estadios larvales $(H=91,54 ; p<0,0001$ y $H=345,41 ; p<0,0001$, respectivamente). Sin embargo, a partir del cambio de larva IV a larva V no se observaron diferencias significativas para el ancho de cápsula cefálica $(p>0,05)$. Tampoco las hubo para la variable largo de la larva a partir del estadio de larva VI $(p>0,05)$. La tasa de incremento para el ancho de las cápsulas cefálicas no presentó una progresión geométrica (Tabla 2).

La fase pupal duró aproximadamente 16 días, lo que resulta similar a lo informado por Hosking y Bain (1977) para individuos $A$. ferus emergidos de árboles de pino a $21^{\circ} \mathrm{C}$ y por Van Epenhuijsen et al. (2012) para miembros de la misma especie cria- 
dos con dieta artificial a $20{ }^{\circ} \mathrm{C}$ (desarrollada por Rogers et al., 2002).

El peso promedio de las pupas hembra $(339,98 \pm 4,21 \mathrm{mg})$ fue estadísticamente superior $(H=11,42 ; N=21 ; p=0,0007)$ que el de las pupas macho $(290,41 \pm 4,47 \mathrm{mg})$ Esta misma diferencia $(\mathrm{N}=21 ; \mathrm{H}=7,16 ; \mathrm{p}=0,0040)$, se encontró al comparar el largo de las pupas de ambos sexos (2,40 \pm $0,02 \mathrm{~cm}$ para las hembras y $2,30 \pm 0,02 \mathrm{~cm}$ para los machos).
A $25^{\circ} \mathrm{C}$ se registraron diferencias significativas en la supervivencia de los estados y estadios larvales de $A$. syriacus $(p<0,01)$ (Figuras 4 y 5$)$. El estado con mayor mortalidad fue el de larva, lo que fue evidente particularmente en el I estadio, donde $60 \%$ de los individuos no lograron sobrevivir. Por otra parte, los estadios II y III presentaron una disminución de sobrevida de 25\%; esto concuerda con las observaciones de Hosking y Bain (1977) para $A$. ferus, donde la mortalidad de larvas está

Tabla 2. Límite inferior (LI), límite superior (LS) y promedio \pm E.E. del ancho de la cápsula cefálica (mm) y largo de la larva (cm) de los estadios larvales de A. syriacus. La tasa de incremento se calculó para el ancho de la cápsula cefálica.

\begin{tabular}{|c|c|c|c|c|c|c|c|}
\hline \multirow{2}{*}{$\begin{array}{l}\text { Estadio } \\
\text { Larval }\end{array}$} & \multicolumn{4}{|c|}{ Ancho de cápsula cefálica (mm) } & \multicolumn{3}{|c|}{ Largo de la larva $(\mathrm{cm})$} \\
\hline & LI & LS & Media \pm E.E. & $\begin{array}{c}\text { Tasa de } \\
\text { incremento }\end{array}$ & $\mathrm{LI}$ & LS & Media \pm E.E. \\
\hline 1 & 1,00 & 2,00 & $1,13 \pm 0,12 a^{1}$ & - & 0,10 & 1,50 & $0,32 \pm 0,04 a$ \\
\hline 2 & 1,50 & 3,50 & $2,64 \pm 0,12 a$ & 2,34 & 0,60 & 2,70 & $1,39 \pm 0,05 b$ \\
\hline 3 & 2,50 & 5,00 & $3,20 \pm 0,12 a$ & 1,21 & 0,60 & 2,80 & $1,77 \pm 0,03 c$ \\
\hline 4 & 3,00 & 5,00 & $4,05 \pm 0,12 b$ & 1,26 & 1,20 & 2,90 & $2,11 \pm 0,05 d$ \\
\hline 5 & 3,50 & 5,00 & $4,02 \pm 0,12 b$ & 0,99 & 1,70 & 3,00 & $2,19 \pm 0,04 d$ \\
\hline 6 & 3,50 & 5,00 & $4,25 \pm 0,14 b$ & 1,05 & 1,60 & 3,20 & $2,49 \pm 0,04 \mathrm{e}$ \\
\hline 7 & 4,00 & 4,00 & $4,00 \pm 0,23 b$ & 0,94 & 2,00 & 2,9 & $2,40 \pm 0,05 e$ \\
\hline 8 & 3,50 & 3,50 & $3,50 \pm 0,40 a b$ & 0,87 & 2,10 & 2,90 & $2,57 \pm 0,07 \mathrm{e}$ \\
\hline 9 & - & - & - & - & 2,00 & 2,60 & $2,36 \pm 0,16 \mathrm{de}$ \\
\hline
\end{tabular}

1. Medias con una letra minúscula común no son significativamente diferentes entre estadios larvales $(p>0,05)$.

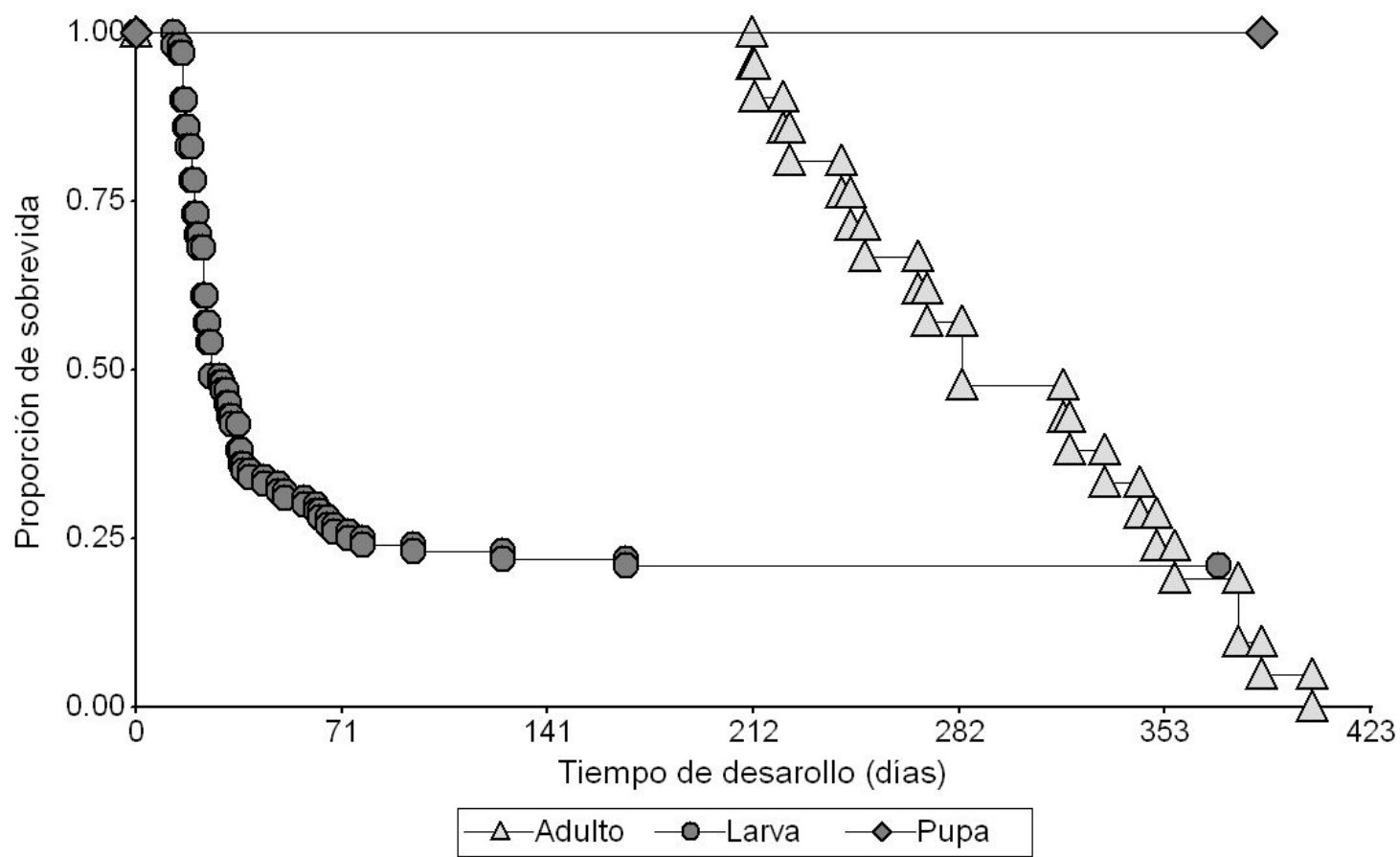

Figura 4. Proporción de supervivencia respecto al tiempo de desarrollo total (días) discriminando los estados de huevo, larva, pupa y adulto de $A$. syriacus. 


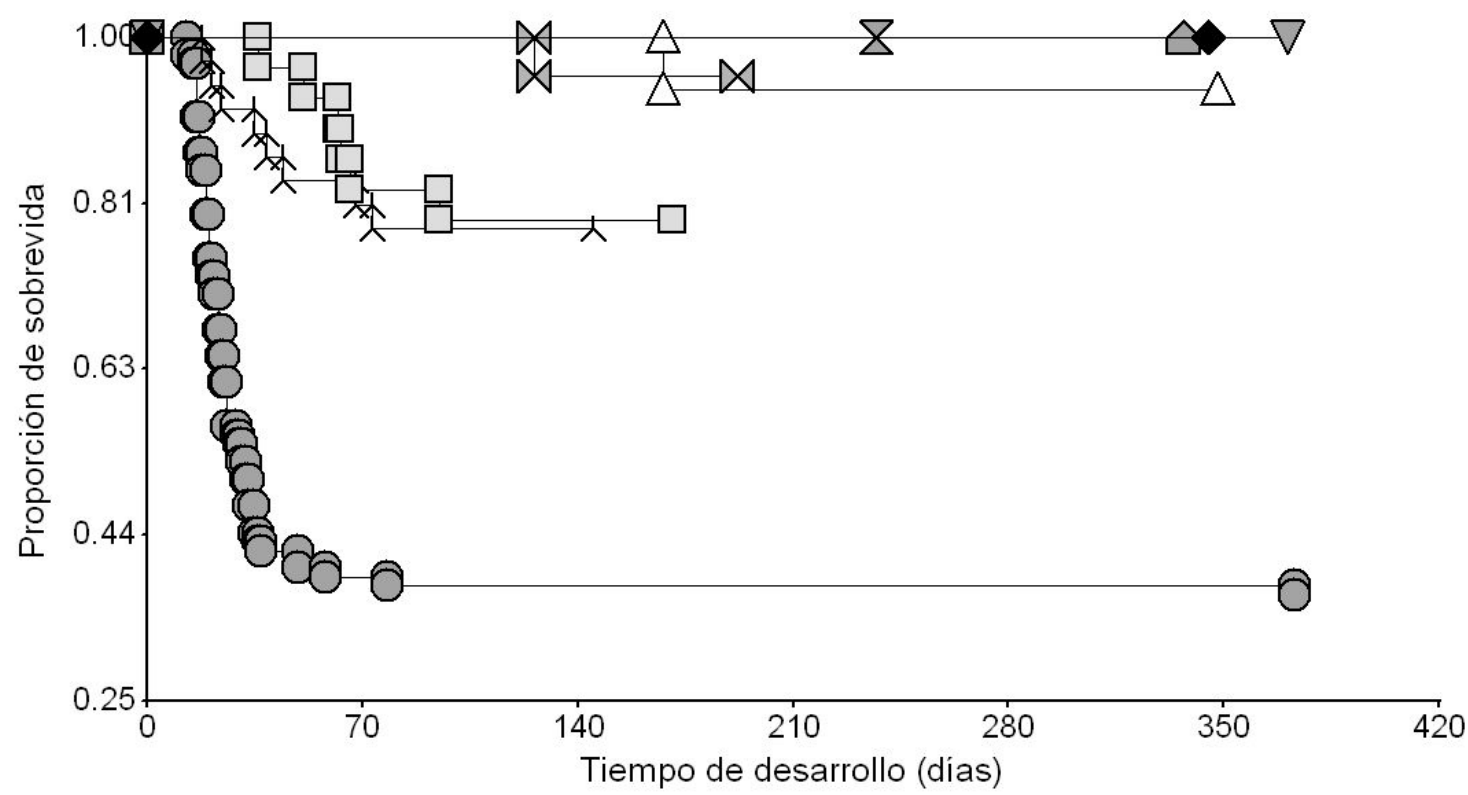

\begin{tabular}{|c|c|c|c|c|c|}
\hline -o-Larva1 & 入 Larva2 & - - Larva3 & $\pitchfork$-Larva4 & $\boldsymbol{z}$ Larva5 & $\nabla$-Larva6 \\
\hline$\triangle$ Larva7 & $\Delta$-Larva8 & —Larva9 & & & \\
\hline
\end{tabular}

Figura 5. Proporción de supervivencia respecto al tiempo de desarrollo total (días) discriminando estadios larvales de A. syriacus.

asociada a los primeros estadios. El $4 \%$ de los individuos del IV estadio no llegaron a desarrollarse. En etapas avanzadas del ciclo, el estadio larval VII fue el más susceptible, aunque la caída de sobrevida no superó el $6 \%$. Los demás estadios larvales finales (Larva 6, 8 y 9) presentaron una supervivencia máxima (100\%) (Fig. 5). Esta misma condición se cumplió para el estado de pupa (Fig. 4). Por último, la proporción de supervivencia de los adultos llegó a tener una caída del 50\% en etapas intermedias de su desarrollo (Fig. 4).

Los datos de tiempo de desarrollo y supervivencia del ciclo de vida completo de $A$. syriacus aquí presentados pueden contribuir al desarrollo de modelos termodependientes y resultan básicos para estudios de dinámicas poblacionales necesarios a la hora de tomar decisiones de manejo.

\section{AGRADECIMIENTOS}

El presente trabajo se desarrolló con la financiación del Proyecto SAFO 111 (Unidad Para el Cambio Rural, Ministerio de Agricultura, Ganadería y Pesca) Proyecto BIRF 7520.

\section{BIBLIOGRAFÍA}

Altman, D.G., 1991. Practical statistics for medical research. Chapter 13: Survival times. Chapman and Hall, $611 \mathrm{pp}$.

Bense, U., 1995. Longicorn beetles: illustrated key to the Cerambycidae and Vesperidae of Europe. Weikersheim: Margraf, $512 \mathrm{pp}$.

Brockerhoff, E.G. and G.P. Hosking, 2001. Arhopalus tristis (F.) (Coleoptera: Cerambycidae). Burnt pine longhorn. New Zealand Forest Service, Forest and Timber Insects in New Zealand 27:1-8.

Brown, F.G., 1968. Pests and diseases of forest plantation trees. Clarendon Press, Oxford, 1330 pp.

Cerezke, H.F., 1977. Characteristics of damage in treelength white spruce logs caused by the white-spotted sawyer, Monochamus scutellatus. Canadian Journal of Forest Research 7(2): 232-240

Ciesla, W., 2011. Forest Entomology: A global perspective. Blackwell Publishing Ltd., 416 pp.

Coulson, R. y J. Witter, 1990. Entomología Forestal: ecología y control. Editorial Limusa S.A. de C.V., México, D.F., $622 \mathrm{pp}$.

Di lorio, O.R., 2004. Especies exóticas de Cerambycidae (Coleoptera) introducidas en Argentina. Parte 2. Nue- 
vos registros, plantas huéspedes, periodos de emergencia, y estado actual. Agrociencia 38: 663-678.

Duffy, E.A.J., 1963. A Monograph of the immature stages of Australasian timber beetles (Cerambycidae). British Museum (Natural History), London, 235 pp.

Duffy, E.A.J., 1968. A monograph of the immature stages of Oriental timber beetles (Cerambycidae). British Museum (Natural History), London, 434 pp.

Esperk, T., 2006. Larval instar as a key element of insect growth schedues. University of Tartu, Faculty of Biology and Geography, $186 \mathrm{pp}$

Esperk, T., T. Tammaru and S. Nylin, 2007. Intraspecific variability in number of larval instars in insects. Journal of Economic Entomology 100(3): 627-645.

Fachinetti, R., 2012. Dinámica poblacional de la oruga medidora Rachiplusia nu (Gueneé) (Lepidoptera: Noctuidae). Tesina de grado - Escuela de Biología Facultad de Ciencias Exactas Físicas y Naturales Universidad Nacional de Córdoba, Argentina, 49 pp.

Fischer, K. and K. Fiedler, 2001. Dimorphic growth patterns and sex-specific reaction norms in the butterfly Lycaena hippothoe sumadiensis. Journal of Evolutionary Biology 14: 210-218.

Gomi, T., 1996. A mechanism for the decrease in developmental period of a trivoltine population of Hyphantria cunea (Lepidoptera: Arctiidae). Applied Entomology and Zoology 31: 217-223.

Gomi, T.; M. Inudo and D. Yamada, 2003. Local divergence in developmental traits within a trivoltine area of Hyphantria cunea Drury (Lepidoptera: Arctiidae). Ento- mological Science 6: 71-75.

Hosking, G.P. and J. Bain, 1977. Arhopalus ferus (Coleoptera: Cerambycidae); its biology in New Zealand. New Zealand Journal Forest Science 7: 3-15.

Hutcheson, J.A., 1980. Arhopalus ferus (Coleoptera: Cerambycidae): structure and function of the female reproductive system. New Zealand Journal of Zoology 7(3): 417- 424.

Iglesias, C.; A. Notario y J.R. Baragaño, 1989. Evaluación de las condiciones de cría y datos bionómicos de coleópteros lignícolas de tocón de pino. Boletín de Sanidad Vegetal Plagas 15: 9-16

Iglesias, M. 2009. Los insectos saproxílicos en la Península Ibérica: Qué sabemos y qué nos gustaría hacer. Boletín Sociedad Entomológica Aragonesa 44: 505512.

Keena, M.A. and P.M. Moore, 2010. Effects of temperature on Anoplophora glabripennis (Coleoptera: Cerambycidae) larvae and pupae. Environmental Entomology 39(4): 1323-1335

Larsson, F.K., 2010. Limb amputation by male Neotropical longhorn beetles during competition for females. Biota Neotropica 10 (1): 339-341.
López, A.; J. García, M. Demaestri, O. Di lorio and R. Magris, 2008. The genus Arhopalus Serville, 1834 (Insecta: Coleoptera: Cerambycidae: Aseminae) in association to Sirex noctilio in Argentina. Boletín de Sanidad Vegetal Plagas 34(4): 529-531.

Mahibbur, R.M. and Z. Govindarajulu, 1997. A modification of the test of Shapiro and Wilks for normality. Journal of Applied Statistics 24(2): 219-235.

Martelli, A., 1952. Sulla presenza in Toscana del Criocephalus (Cephalocrius) syriacus Reitt. Redia Giornale di Zoologia 37: 479-486.

Minelli, A., 1993. Biological systematics. Chapman \& Hall, London, $386 \mathrm{pp}$

Moore, K.M., 1962. Insect attack on Pinus spp. Forestry Commission of New South Wales Research Note No. 12, pp. 1-14.

Moore, K.M., 1963. Observations on some Australian forest insects. 14. A preliminary list of insects attacking Pinus spp. in New South Wales. Australian Zoologist 13: $69-77$

Naves, P.M.; E. Sousa, J.M. Rodrigues, 2008. Biology of Monochamus galloprovincialis (Coleoptera: Cerambycidae) in the pine wild disease affected zone, Southern Portugal. Silva Lusitana 16(2): 133-148.

Pedemonte, M.L., 2014. Efecto de la conectividad del paisaje, el tamaño y la calidad del parche sobre la abundancia poblacional de dos especies de insectos herbívoros. Tesis, Universidad Nacional de Córdoba, Argentina, $128 \mathrm{pp}$.

Pershing, J.C. and M.J. Linit, 1986. Development ans seasonal ocurrence of Monochamus carolinensis (Coleoptera: Cerambycidae) in Missouri. Environmental Entomology 15: 251-253.

Reitter, E., 1895. Sechster Beitrag zur Coleopteren-Fauna von Europa und den angrenzenden Ländern. Wiener Entomologische Zeitung 14: 199-204.

Robertson, L.; A. García-Álvarez, S.C. Arcos, M.A. DíezRojo, J.P. Mansilla, R. Sanz, C. Martínez, M. Escuer, L. Castresana, A. Notario, A. Bello and M. Arias. 2008. Potential insect vectors of Bursaphelenchus spp. (Nematoda: Parasitaphelenchidae) in Spanish pine forests. In: Mota, M.M. and P. Vieira (Eds): Pine wilt disease: a worldwide threat to forest ecosystems. Dordrecht, The Netherlands, Springer, pp. 221-233.

Rogers, D.J.; S.E. Lewthwaite and P.R. Dentener, 2002. Rearing huhu beetle larvae, Prionoplus reticularis (Coleoptera: Cerambycidae) on artificial diet. New Zealand Journal of Zoology 29:303-310.

Rose, A.H., 1957. Some notes on the biology of Monochamus scutellatus (Say) (Coleoptera: Cerambycidae). The Canadian Entomologist 89(12): 547-553.

Safranyik, L. and A.G. Raske, 1970. Sequential sampling plan for larvae of Monochamus in lodgepole pine logs. 
Journal of Economic Entomology 63: 1903-1906.

van Epenhuijsen, C.W.; K.G. Somerfield and D. Hedderley, 2012. Rearing and storing Arhopalus ferus life stages in the laboratory for experimental purposes. New Zealand Journal of Forestry Science 42: 15-23.

Wallace, H.R., 1954. Notes on the biology of Arhopalus ferus Mulsant (Coleoptera: Cerambycidae). Proceedings of the Royal Entomological Society of London. Series A. General Entomology 29: 99-113.

Wang, Q. and R.A.B. Leschen, 2003. Identification and distribution of Arhopalus species (Coleoptera: Ce- rambycidae: Aseminae) in Australia and New Zealand. New Zealand Entomologist 26(1): 53-59.

Wang, Y.; J.X. Zhen Wang, H. Kim, and C. Sung. 2014. An effective attractant for the pinewood nematode vector Arhopalus rusticus in South Korea. Journal of Entomology and Zoology Studies 2(2): 76-80.

Webb, G.A. and R.H. Eldridge, 1997. Arhopalus syriacus (Reitter) (Coleoptera: Cerambycidae): a potential economic pest of Pinus in Australia, with notes on its biology and distribution. Australian Forestry 60(2): 125-129. 